\title{
Caracterización estructural y óptica de películas delgadas de dióxido de titanio depositadas por rociado pirolítico
}

\section{Structural and optical characterization of titanium dioxide thin films deposited by pyrolytic sputtering.}

\author{
DOI: $10.46932 / \mathrm{sfjdv2n2-079}$
}

Received in: March 1st, 2021

Accepted in: May 30th, 2021

\author{
Ricardo Otilio Verde Vera \\ Departamento de Física y Meteorología, Universidad Nacional Agraria La Molina, Lima, Perú \\ E-mail: ricardovera@lamolina.edu.pe \\ Oscar Enrique Tang Cruz \\ Departamento de Física y Meteorología, Universidad Nacional Agraria La Molina, Lima, Perú \\ E-mail: otang@lamolina.edu.pe \\ Juan Manuel Pesantes Rojas \\ Departamento de Física y Meteorología, Universidad Nacional Agraria La Molina, Lima, Perú \\ E-mail: jmpesantes@ lamolina.edu.pe \\ Eusebio Idelmo Cisneros Tarmeño \\ Departamento de Física y Meteorología, Universidad Nacional Agraria La Molina, Lima, Perú \\ ecisneros@lamolina.edu.pe \\ Julio Alfonso Arakaki Kiyán \\ Departamento de Física y Meteorología, Universidad Nacional Agraria La Molina, Lima, Perú \\ E-mail: jarakaki@lamolina.edu.pe \\ Hernan Oscar Cortez Gutierrez \\ Departamento de Educación Física-Facultad ciencias de la salud \\ Universidad Nacional del Callao, Callao, Perú \\ E-mail: hernanoscarcortezgutierrez@gmail.com \\ Milton Milciades Cortez Gutierrez \\ Departamento de Matemática \\ Universidad Nacional de Trujillo, Trujillo, Perú \\ E-mail: mcortezgutierrez@yahoo.es
}

\section{RESUMEN}

Se sintetizó películas de dióxido de titanio $\left(\mathrm{TiO}_{2}\right)$ por rociado pirolítico, mediante una solución precursora de diisopropóxido de titanio estabilizado con acetil-acetonato al $75 \%$ en isopropanol disuelto en metanol. Esta solución se roció sobre sustratos de vidrio en una cámara de reacción a 30 psi de presión a diferentes temperaturas $\left(380,430\right.$ y 480 y 380 recocida a $\left.500{ }^{\circ} \mathrm{C}\right)$. La difracción de rayos X (DRX), estudió, el tamaño de grano, microtensión y parámetros de red. La espectroscopia UV-Vis, estudió la transmitancia, índice de refracción, espesor de las películas y ancho de banda prohibida. El equipo de cuatro puntas colineales estudió la relación voltaje-corriente. A $380{ }^{\circ} \mathrm{C}$, la película es amorfa y las demás presentaron fase anatasa con pico predominante (101). La película crecida a $480{ }^{\circ} \mathrm{C}$ presenta el pico de rutilo (211). 
El tamaño de grano disminuye y la microtensión aumenta con la temperatura. Las películas de 480 y 500 ${ }^{\circ} \mathrm{C}$ muestran regular transparencia en la región visible (50-75\%). El índice de refracción se encuentra el rango de 1,96-2,43 y el ancho de banda prohibida tiene aproximadamente 3,2 eV. La gráfica voltaje corriente, es lineal para las muestras crecidas a $480{ }^{\circ} \mathrm{C}$.

Palabras clave: dióxido de titânio, películas delgadas, rociado pirolítico, difracción, energía de banda prohibida.

\begin{abstract}
Titanium dioxide (TiO2) films were synthesized by pyrolytic sputtering using a precursor solution of titanium diisopropoxide stabilized with $75 \%$ acetyl-acetonate in isopropanol dissolved in methanol. This solution was sprayed onto glass substrates in a reaction chamber at 30 psi pressure at different temperatures (380, 430 and 480 and 380 annealed at $500{ }^{\circ} \mathrm{C}$ ). X-ray diffraction (XRD), studied, grain size, microtension and lattice parameters. UV-Vis spectroscopy studied transmittance, refractive index, film thickness and forbidden bandwidth. Collinear four-prong equipment studied the voltage-current relationship. At $380{ }^{\circ} \mathrm{C}$, the film is amorphous and the others presented anatase phase with predominant peak (101). The film grown at $480{ }^{\circ} \mathrm{C}$ shows rutile peak (211). The grain size decreases and the microtension increases with temperature. The 480 and $500{ }^{\circ} \mathrm{C}$ films show regular transparency in the visible region (50-75\%). The refractive index is in the range of 1.96-2.43 and the forbidden bandwidth is approximately $3.2 \mathrm{eV}$. The voltage-current plot is linear for the samples grown at $480{ }^{\circ} \mathrm{C}$.
\end{abstract}

Keywords: titanium dioxide, thin films, pyrolytic sputtering, diffraction, bandgap energy.

\title{
1 INTRODUCCIÓN
}

La tecnología de construcción de dispositivos electrónicos ha permitido el desarrollo de nuevos materiales, con propiedades mejoradas, para diversas aplicaciones en la industria especialmente la electrónica. Es así que ha surgido interés por el estudio, fabricación y caracterización de películas delgadas; las cuales pueden fabricarse de diversos materiales incluyendo metales, óxidos metálicos y sustancias orgánicas. Dichas películas son usadas como componentes fundamentales con diversa funcionalidad, tales como aislantes, barreras de difusión, capacitores dieléctricos, a veces también como electrodos conductores y componentes piezoeléctricos, etc. Estos componentes son de especial importancia para la construcción de circuitos integrados, los cuales son la tecnología básica para memorias semiconductoras y de tipo LSI versátiles (Flash, DRAM, SRAM, RRAM, etc). Ouyang (2016) argumenta que los RRAM son los candidatos prometedores para las memorias de la próxima generación. El tamaño puede reducirse a menos de $10 \mathrm{~nm}$ y su tiempo de respuesta puede alcanzar nanosegundos (ns).

Diversos tipos de materiales desde óxidos simples (tales como $\mathrm{SiO}_{2}, \mathrm{AL}_{2} \mathrm{O}_{3}, \mathrm{TiO}_{2}, \mathrm{ZnO}, \mathrm{ZrO}_{2}$, $\mathrm{CeO}_{2}$, etc.) hasta óxidos complejos $\left(\mathrm{SrTiO}_{3}, \mathrm{In}_{2} \mathrm{O}_{3}-\mathrm{SnO}_{2}, \mathrm{SrRuO}_{3}\right.$, etc.) son procesados para ser componentes de películas delgadas en obleas de silicio ( $\mathrm{Si}$ ). Por otro lado, en su investigación (Kumar et al. 2016) concluyó que los estudios también indican que las películas delgadas son candidatos prometedores para celdas solares. Así mismo, en su investigación (Franco, 2018) concluye que el depósito 
de un film $\mathrm{TiO}_{2}$ sobre diversos sustratos permite aumentar la dureza y la resistencia al desgaste de sus superficies, además si el film crece en fase rutilo mejora su biocompatibilidad y hemocompatibilidad tornando estos recubrimientos de interés para el desarrollo de biomateriales. El término película delgada se refiere a películas cuyo espesor va de $0,1 \mu \mathrm{m}$ hasta unos $300 \mu \mathrm{m}$. $\mathrm{TiO}_{2}$ generalmente se puede encontrar en tres formas alotrópicas diferentes a presión atmosférica: rutilo (tetragonal, grupo espacial P42/mnm), anatasa (tetragonal, grupo espacial I4/1/amd) y brookita (ortorrómbica, grupo espacial Pcab) (Lee et al., 2002). Cada una de las fases la hacen útil para diferentes aplicaciones.

Las películas delgadas de $\mathrm{TiO}_{2}$ son muy usadas por sus propiedades especiales: buena transmisión en las regiones del espectro visible y cerca al infrarrojo, buena adhesión y alta estabilidad contra la abrasión mecánica, ataque químico y altas temperaturas (Ritter, 1975; Pulker, 1984). Las películas de $\mathrm{TiO}_{2}$ pueden ser fabricadas usando diferentes técnicas. La técnica de rociado pirolítico es una técnica de fabricación sencilla y de bajo costo. Entre las películas delgadas más interesantes procesadas por esta técnica están las películas de $\mathrm{TiO}_{2}$.

En esta investigación se sintetizó películas delgadas de $\mathrm{TiO}_{2}$ mediante rociado pirolítico y se caracterizaron para verificar sus propiedades morfológicas, ópticas y eléctricas utilizando difracción de rayos X, espectrofotometría de absorción Ultravioleta - Visible (UV-Vis) y técnicas de medición de la resistividad eléctrica respectivamente.

\section{MATERIALES Y MÉTODOS}

Las películas delgadas de dióxido de titanio $\left(\mathrm{TiO}_{2}\right)$ fueron fabricadas por rociado pirolítico a partir de una solución de diisopropóxido de Titanio (reactivo precursor) estabilizado con acetil acetonato al 75 $\%$ en isopropanol disuelto en metanol, y depositadas en substratos de vidrio de 76x26x1 mm de dimensión.

\subsection{ESTRUCTURA CRISTALINA}

La determinación de la estructura cristalina de las películas de $\mathrm{TiO}_{2}$ se hizo mediante el análisis de los espectros de difracción de rayos $\mathrm{X}$ que se obtuvieron usando un equipo marca BRUKER, D8 ADVANCED ECO (Figura 1a). Para identificar la fase de la estructura cristalina de las películas de $\mathrm{TiO}_{2}$, se compararon los datos del difractograma obtenido con la base de datos ICSD - 1997 del equipo. Los barridos fueron realizados con ángulos de $2 \theta$, con valores entre 15 y $70^{\circ}$, con un paso típico de $0,02^{\circ}$.

La distancia interplanar (d), determinó usando la siguiente ecuación 1 (Ley de Bragg):

$$
\operatorname{sen} \theta=\frac{\lambda}{2 d_{h k l}}
$$


Donde $\lambda$ es la longitud de onda de los rayos $\mathrm{X}, \lambda=0,15418 \mathrm{~nm}$ y $\square$ es el ángulo del pico de máxima intensidad.

El tamaño de grano (D), fue obtenido mediante la ecuación 2:

$$
\mathrm{D}=\frac{\mathrm{k} \lambda}{\beta_{\mathrm{hkl}} \cos \theta}
$$

donde k toma el valor de 0,90; $\square \square$ es el ancho medio del pico máximo de rayos $\mathrm{X}$ (FWHM). debe tomarse en radianes. 
Figura 1. (a) Difractómetro de rayos X (XRD) de la marca BRUKER, Modelo D8 ADVANCED ECO con Cátodo de Cu $-\alpha$ $(\lambda=0,15418 \mathrm{~nm})$. (b) Espectrofotómetro UV-Vis (Thermo Scientific - Modelo Evolution 600; A = $190-1100 \mathrm{~nm}$ ).
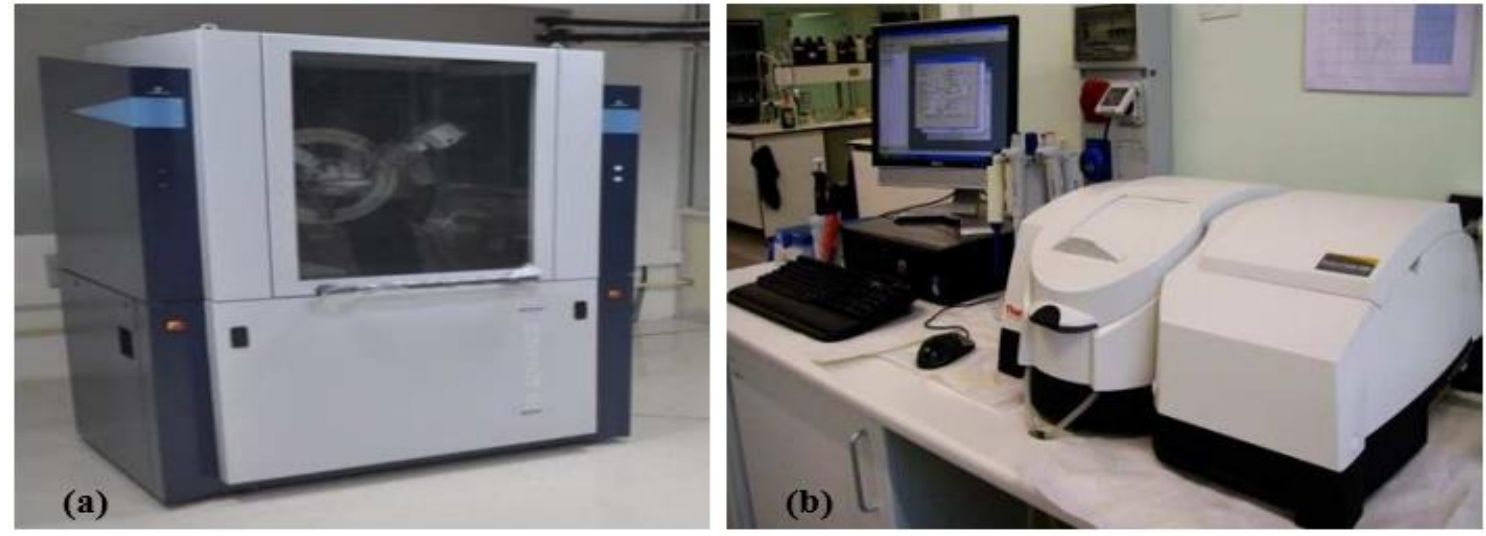

Los parámetros de red: a, b y c; en los que se cumple que a = b, siendo c diferente; pues el titanio tiene una estructura tetragonal (tanto en anatasa como en rutilo), se obtuvieron con la ecuación 3.

$$
\frac{1}{d_{h k l}^{2}}=\left[h^{2}+k^{2}+l^{2}\left(\frac{a}{c}\right)^{2}\right] \frac{1}{a^{2}}
$$

por otro lado, la microtensión $(\square)$ se calculó utilizando la siguiente ecuación (Cullity, 1978):

$$
\varepsilon=\left(\frac{1}{\operatorname{sen} \theta}\right)\left[\left(\frac{\lambda}{D}\right)-(\beta \cos \theta)\right]
$$

Además, la densidad de dislocación $(\delta)$ se calculó usando la siguiente ecuación (Mahoharan y Sridhar, 2012):

$$
\delta=\frac{1}{\mathrm{D}^{2}}
$$

Así mismo la densidad de la celda unitaria ( $\square$ ) para la anatasa usando la siguiente ecuación (Scherrer, 1918):

$$
\rho=\frac{4 \times M}{N \times a^{2} \times c} g \cdot \mathrm{cm}^{-3}
$$

donde "M" es la masa molecular del $\mathrm{TiO}_{2}(79,87 \mathrm{~g} / \mathrm{mol}), \mathrm{N}$ es el número de Avogadro $\left(6,022 \times 10^{23}\right.$ entidades/mol), finalmente a y c son los parámetros de red en $\mathrm{cm}$. 


\subsection{PROPIEDADES ÓPTICAS}

La transmitancia y absorbancia de la película de $\mathrm{TiO}_{2}$ se obtuvieron con el espectrofotómetro de absorción Ultravioleta y Visible (UV - Vis) de marca Thermo Scientific, Modelo Evolution 600; A=1901100 nm (Figura 1b). El índice de refracción (n) y espesor de las películas (t) se encontraron obteniendo los valores de transmitancia máxima ( $\mathrm{T}_{\mathrm{MÁx}}$ ) y mínima $\left(\mathrm{T}_{\text {mín }}\right)$ del espectro de transmisión. Es posible calcular el índice de refracción de la película, $\mathrm{n}_{\mathrm{p}}$, teniendo en cuenta que el índice de refracción del sustrato, en este caso vidrio, es $\mathrm{n}_{\mathrm{s}}=1,5 \mathrm{y}$ el del medio en que se encuentran las muestras, en este caso aire, es $\mathrm{n}_{\mathrm{a}}=1$. El índice de refracción, así como el espesor de las películas delgadas fueron determinados utilizando un método óptico conocido como el modelo de Goodman. El modelo (Goodman, 1978) propone que el índice de refracción se puede calcular a partir de la ecuación:

$$
\begin{gathered}
\mathrm{n}_{\mathrm{p}}=\left\{\frac{-\left(\mathrm{n}_{\mathrm{a}}^{2}+\mathrm{n}_{\mathrm{s}}^{2}\right)\left(1-2 \rho_{\mathrm{T}_{2}}\right)+\left[\left(\mathrm{n}_{\mathrm{a}}^{2}+\mathrm{n}_{\mathrm{s}}^{2}\right)^{2}\left(1-2 \rho_{\mathrm{T}_{2}}\right)^{2}-4 \mathrm{n}_{\mathrm{a}}^{2} \mathrm{n}_{\mathrm{s}}^{2}\right]^{\frac{1}{2}}}{2}\right\}^{\frac{1}{2}} \\
\rho_{\mathrm{T}_{2}}=\frac{\mathrm{T}_{\mathrm{MÁx}}}{\mathrm{T}_{\text {mín }}}
\end{gathered}
$$

Es posible conocer el espesor t de la película, a partir de los valores del índice de refracción de la película $\mathrm{n}_{\mathrm{p}}$, longitud de onda correspondientes a la transmitancia máxima y mínima y conociendo el ángulo de incidencia de la luz con respecto a la película; mediante la expresión:

$$
\mathrm{t}=\frac{\mathrm{M}_{\mathrm{ab}} \lambda_{\mathrm{a}} \lambda_{\mathrm{b}}}{2\left(\lambda_{\mathrm{a}}-\lambda_{\mathrm{b}}\right)\left(\mathrm{n}_{\mathrm{p}}^{2}-\operatorname{sen}^{2} \theta_{0}\right)^{1 / 2}}
$$

dónde: $\lambda_{\mathrm{a}} \mathrm{y} \lambda_{\mathrm{b}}$ corresponden a los valores de longitud de onda para la transmitancia máxima y mínima respectivamente, mientras que $\mathrm{M}_{\mathrm{ab}}$ es el número de máximos o mínimos que separan a $\lambda_{\mathrm{a}}$ de $\lambda_{\mathrm{b}}$; y $\theta_{0}$ el ángulo incidente de luz.La porosidad (P) se obtiene usando la relación de Lorenz-Lorentz (Mezza et al.,1999).

$$
P=1-\frac{n^{2}-1}{n^{2}+2} \cdot \frac{n_{b}^{2}+2}{n_{b}^{2}-1}
$$


donde $\mathrm{n}_{\mathrm{b}}$ es el índice de refracción del $\mathrm{TiO}_{2}$ fase anatasa libre de porosidad $\left(\mathrm{n}_{\mathrm{b}}=2,49\right)$ y $\mathrm{n}$ es el índice de refracción de las películas.

\subsection{ANCHO DE BANDA PROHIBIDA $\left(\mathrm{E}_{\mathrm{G}}\right)$}

Para determinar el valor de $\mathrm{E}_{\mathrm{g}}$; se partió del gráfico (Fig. 5a) de (hv* $\left.\square\right)^{1 / 2}$ como una función la energía del fotón $(h v)$, h es la constante de Planck, $v$ es la frecuencia de la radiación y $\square$, conocido como el coeficiente de absorción que se determinó usando siguiente ecuación:

$$
\alpha=\frac{2.3}{\mathrm{t}} \log \left(\frac{\mathrm{I}_{0}}{\mathrm{I}}\right)
$$

donde $\mathrm{t}$ es el espesor de la muestra, $\mathrm{I}_{0}$ es la intensidad que atraviesa una película delgada terminando con una intensidad I. Usando el modelo Tauc (Tauc et al., 1966), para transiciones indirectas se realizó la regresión lineal en la zona más recta de la curva, obteniendo la ecuación de ésta, donde la extrapolación nos permite obtener el valor del ancho de banda prohibida con los datos de la pendiente $(\mathrm{m})$ y la ordenada al origen (b) de la ecuación. Por lo tanto, $E_{g}=-b / m$, como se muestra en la figura 5a. El valor de $n=1 / 2$, el cual es un valor característico para la transición permitida indirecta, la cual domina sobre toda la absorción óptica de películas delgadas de $\mathrm{TiO}_{2}$ (Mardare et al., 2000; Tang et al., 1994). Para las películas fabricadas a $380{ }^{\circ} \mathrm{C}, 430{ }^{\circ} \mathrm{C}, 480{ }^{\circ} \mathrm{C}$ y $380{ }^{\circ} \mathrm{C}$ recocida a $500{ }^{\circ} \mathrm{C}$ se obtuvieron energías de banda prohibida de 3,234 eV, 3,210 eV, 3,196 eV y 3,204 eV respectivamente (figura 5b). Para determinar el valor de $\mathrm{E}_{\mathrm{g}}$; se asumió que $(\mathrm{h} v * \square)^{1 / 2}=0$, por lo tanto, $\mathrm{E}_{\mathrm{g}}=-\mathrm{b} / \mathrm{m}$, como se muestra en la figura $5 \mathrm{~b}$ donde se muestran la pendiente $(\mathrm{m})$ y la ordenada al origen (b) de la ecuación. Para las películas fabricadas a $380{ }^{\circ} \mathrm{C}, 430{ }^{\circ} \mathrm{C}, 480{ }^{\circ} \mathrm{C}$ y $380{ }^{\circ} \mathrm{C}$ recocida a $500{ }^{\circ} \mathrm{C}$ se obtuvieron energías de banda prohibida de $3,234 \mathrm{eV}, 3,210 \mathrm{eV}, 3,196 \mathrm{eV}$ y 3,204 eV respectivamente (figura 5b).

\section{RESULTADOS Y DISCUSIÓN}

La formación de películas fue producida a través de descomposición térmica del precursor sometido a oxígeno:

$$
\mathrm{C}_{16} \mathrm{H}_{28} \mathrm{O}_{6} \mathrm{Ti}+21 \mathrm{O}_{2} \rightarrow \mathrm{TiO}_{2}+16 \mathrm{CO}_{2}+14 \mathrm{H}_{2} \mathrm{O}
$$

Esto conduce a películas incoloras y claras, mientras que las películas obtenidas en la ausencia de oxígeno contienen carbono, tal como sucede mediante el método de fabricación de películas delgadas por Aerosol Pyrolysis de un precursor orgánico metálico similar (Shinde et al., 2008). 


\subsection{DIFRACTOGRAMAS DE LAS PELÍCULAS DELGADAS DE DIÓXIDO DE TITANIO}

La Difracción de Rayos X mostró que la película depositada a temperatura de sustrato de $380{ }^{\circ} \mathrm{C}$ es totalmente amorfa y no muestra pico de difracción de $\mathrm{TiO}_{2}$ (curva de color negro en Fig. 3a). Pero, al someterla al recocido hasta $500{ }^{\circ} \mathrm{C}$ por de 2 horas, se presenta la fase cristalina anatasa, tal como lo muestran los picos de difracción (101), (004), (200) y (211) detectados claramente en la gráfica (color rojo en fig. 2a). Los picos mencionados corresponden a los ángulos de difracción de 25,277 ; 37,884 ${ }^{\circ}$; $48,019^{\circ}$ y $54,990^{\circ}$ respectivamente.

Figura 2. (a)Muestras depositadas a $380{ }^{\circ} \mathrm{C}$ sin recocido (curva negra) y con recocido a $500{ }^{\circ} \mathrm{C}$ por espacio de dos horas (curva roja). La muestra recocida presenta fase anatasa de $\mathrm{TiO}_{2}$. (b)Patrón de difracción de rayos $\mathrm{X}$ películas delgadas de $\mathrm{TiO}{ }_{2}$ depositadas y preparadas a temperaturas de $380{ }^{\circ} \mathrm{C}, 430{ }^{\circ} \mathrm{C}$ y $480{ }^{\circ} \mathrm{C}$ sin recocido.
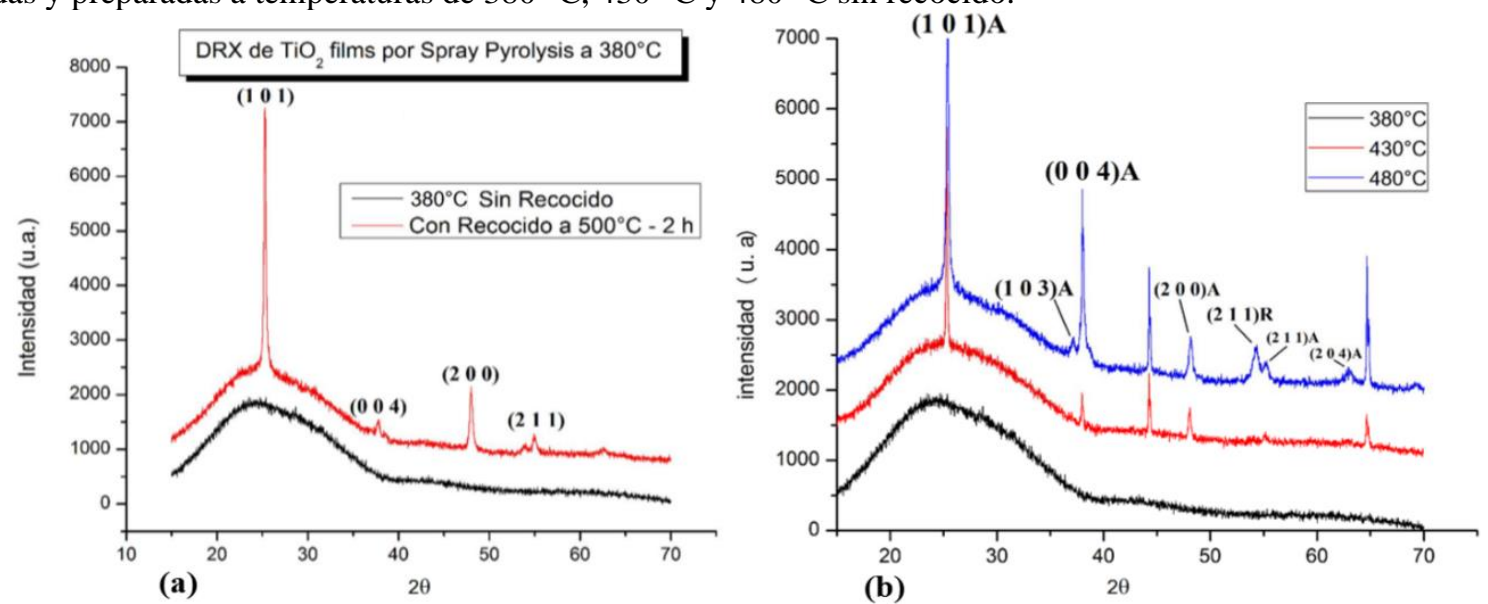

Las películas depositadas a $430{ }^{\circ} \mathrm{C}$ (color rojo en la figura 2 b) muestran presencia de $\mathrm{TiO}_{2}$ en fase anatasa en su composición, según los planos (101), (004), (200) y (211), siendo (101) el predominante. Las películas depositadas a $480{ }^{\circ} \mathrm{C}$ (Color azul en la figura 2b) tienen también presencia de anatasa en los planos de difracción (101), (103), (004), (200), (211), (204) pero además muestran el pico (211) R que pertenece, sin lugar a dudas, a la fase rutilo según DRX. Con el aumento de la temperatura de deposición, las películas mostraron una mayor cristalinidad, que se manifiesta por el aumento correspondiente en la intensidad de los picos en difractogramas. (Gapale et al., 2018).

Además, mientras se aumenta la temperatura de deposición y/o de recocido se promueve la aparición de picos característicos de fase rutilo, la cual suele formarse como fase única a temperaturas de recocido por encima de $\operatorname{los} 700{ }^{\circ} \mathrm{C}$ (Bartic et al., 2013). Pero a pesar que las películas depositadas a 480 ${ }^{\circ} \mathrm{C}$ revelan dos picos adicionales de anatasa (103) en $37,148^{\circ}$ y (204) en $62,914^{\circ}$ respectivamente, y revelan un pico de rutilo (211) en 54,209 ․ El pico depende de los parámetros de deposición de las películas fabricadas en el presente trabajo. Esto no es sorpresa puesto que para (Escobar et al., 1999), por el método de ablación láser y (Agudelo et al., 2014) por el método de arco catódico; es posible la formación de películas que revelan presencia de rutilo a temperatura de sustrato incluso menor a $300{ }^{\circ} \mathrm{C}$, 
y así comienza la aparición de un estado de transición de fase anatasa-rutilo que de acuerdo con la literatura se da generalmente a partir de $600{ }^{\circ} \mathrm{C}$ (Agudelo et al., 2014).

Los picos no señalados en la gráfica no coinciden con ningún pico del patrón de $\mathrm{TiO}_{2}$. Estos picos indican presencia de silicatos que forman parte de la estructura del sustrato de vidrio o también presencia de algunas impurezas residuales, producto de la fabricación de películas de otra composición, que no fueron removidas completamente del sustrato y del interior del equipo durante el proceso de limpieza.

En la tabla 1 se muestran la distancia interplanar, tamaño de grano, los parámetros de red, la microtensión, densidad de dislocación y densidad de la anatasa, calculados en función de la temperatura de sustrato de las películas de $\mathrm{TiO}_{2}$.

Las distancias interatómicas calculadas están en el orden de los valores reportados por (Hanini et $a l ., 2013)$ en su trabajo de fabricación de películas por el método de sol-gel. Trabajos anteriores indican que el tamaño de grano de las películas disminuye o aumenta a medida que cambia la temperatura de sustrato. El tamaño de grano de las películas delgadas de $\mathrm{TiO}_{2}$ varían desde 30 hasta $52 \mathrm{~nm}$ aproximadamente en virtud de la temperatura de sustrato, tal como se puede apreciar en la tabla 1. Los valores calculados concuerdan con el orden de los valores reportados por otros autores (Acosta et al., 2005; Shinde et al., 2008; Manoharan y Sridhar, 2012; Hanini et al., 2013). El cálculo de los parámetros de red de la estructura solo se hizo para las películas que mostraron fase cristalina. Estas son de $430{ }^{\circ} \mathrm{C}$, $480{ }^{\circ} \mathrm{C}$ y $380{ }^{\circ} \mathrm{C}$ con recocido a $500{ }^{\circ} \mathrm{C}$. Estos valores están en el orden de valores obtenidos por otros autores anteriormente (Manoharan y Sridhar, 2012; Agudelo et al., 2014).

Tabla 1. Parámetros estructurales de películas delgadas de $\mathrm{TiO}_{2}$ para diferentes temperaturas de sustrato. Posiciones $2 \square\left({ }^{\circ}\right)$ del pico (101). 430,480 y 380 con recocido a $500{ }^{\circ} \mathrm{C}$ para 25,$339 ; 25,400$ y $25,277^{\circ}$ respectivamente.

\begin{tabular}{|c|c|c|c|c|c|c|c|}
\hline $\begin{array}{c}\text { Temperatura } \\
\text { de } \\
\text { sustrato } \\
\left({ }^{\circ} \mathrm{C}\right)\end{array}$ & $\begin{array}{l}\text { Tamaño de } \\
\text { grano D } \\
\text { (nm) }\end{array}$ & $\begin{array}{c}\text { Parámetros } \\
\text { de red a y b } \\
\text { del pico } \\
(101)(\AA)\end{array}$ & $\begin{array}{c}\text { Parámetro } \\
\text { de red c } \\
\text { del pico } \\
(101)(\AA)\end{array}$ & $\begin{array}{c}\square \square \\
\text { FWHM } \\
\text { x10 } \\
\text { (rad) }\end{array}$ & $\begin{array}{l}\text { Micro } \\
\text { tensión } \\
(\square) \\
\times 10^{-4}\end{array}$ & $\begin{array}{c}\text { Densidad } \\
\text { de dislocación } \\
\square \square \times 10^{14} \\
\left(\text { líneas } / \mathrm{m}^{2}\right)\end{array}$ & $\begin{array}{c}\text { Densidad } \\
\text { de } \\
\text { Anatasa } \\
\left(\mathrm{g} / \mathrm{cm}^{3}\right)\end{array}$ \\
\hline 430 & 51,910 & 3,785 & 9,470 & 2,74 & 13,54 & 3,71 & 3,910 \\
\hline 480 & 30,790 & 3,778 & 9,496 & 4,62 & 22,78 & 10,55 & 3,915 \\
\hline $\begin{array}{c}380 \text { recocido } \\
\text { a } 500\end{array}$ & 35,990 & 3,794 & 9,499 & 3,96 & 19,21 & 7,72 & 3,880 \\
\hline
\end{tabular}

En la tabla 1 también es notorio que cuando hay un incremento en la temperatura la microtensión aumenta considerablemente. Este resultado contrasta con el hallado por (Hanini et al., 2013). Pero concuerda con lo reportado por (Manoharan y Sridhar, 2012) en su informe de trabajo de fabricación de películas por el método de rociado pirolítico. El cambio en este tipo de microtensión puede ser debido al proceso de recristalización predominante en las películas delgadas policristalinas. Por otro lado, valores 
reportados de la densidad de dislocación varían con la temperatura tal como reporta (Manoharan y Sridhar, 2012). Además, los datos densidad están en el orden de los valores estándar de dióxido de titanio cristalizado en fase anatasa. En la tabla 1, el ancho total de la mitad del pico máximo FWHM tiene una relación inversa con el tamaño del grano.

Así mismo, se observa que la microtensión y la densidad de dislocación se incrementan a medida que disminuye el tamaño del grano. Las microtensiones que afectan la red de las películas también influyen en los resultados de la espectrometría, produciendo un ensanchamiento de los picos de difracción. Esto es comprobable con los datos de tabla 1, a mayor microtensión, menor tamaño de grano y por ende mayor ensanchamiento del pico de difracción. Esto explica porque la película crecida a $430{ }^{\circ} \mathrm{C}$ tiene el pico predominante (101) más agudo de los tres, dado que experimenta el menor valor de microtensión.

Es importante notar que las películas con recocido a $500{ }^{\circ} \mathrm{C}$ presentan valores que no marcan una tendencia respecto a las otras dos que no fueron recocidas. Se debe tener en cuenta que inicialmente fueron fabricadas a $380{ }^{\circ} \mathrm{C}$, y mediante recocido se cristalizaron, en un proceso de post crecimiento, hasta la temperatura de $500{ }^{\circ} \mathrm{C}$. Podríamos decir que se interrumpió su proceso completo de crecimiento por un lapso de tiempo y luego de enfriarlo hasta la temperatura ambiente fue calentado hasta terminarlo a 500 ${ }^{\circ} \mathrm{C}$. Es probable que este cambio en el proceso de crecimiento sea la base del surgimiento de un nuevo parámetro de fabricación.

\subsection{CURVAS DE TRANSMITANCIA Y ABSORBANCIA}

\subsubsection{Espectros de transmitancia}

La Figura 3(a) muestra los espectros de transmitancia para películas de $\mathrm{TiO}_{2}$ depositadas sobre vidrio en el rango espectral de 300 - $1100 \mathrm{~nm}$. Las curvas muestran una fuerte disminución de la transmitancia a longitudes de onda inferiores a $350 \mathrm{~nm}$ en todos los casos. En el espectro visible, las películas con temperatura de sustrato 380 y $430{ }^{\circ} \mathrm{C}$, muestran una pobre capacidad de transmisión. Pero, ésta se va acrecentando a medida que las películas son expuestas a longitudes de onda mayores, con tendencia hacia el infrarrojo. Se nota una ligera ventaja de transmisión a favor de la película fabricada a $380{ }^{\circ} \mathrm{C}$. Por otra parte, la película con $\mathrm{T}_{\mathrm{s}}=480{ }^{\circ} \mathrm{C}$, tiene mejores indicadores de transmitancia para radiación con longitud de onda entre 360 y $700 \mathrm{~nm}$. Los cual van creciendo gradualmente desde 40 hasta $80 \%$ en el espectro visible, a medida que se acerca al infrarrojo.

La transmitancia de la película crecida a $380{ }^{\circ} \mathrm{C}$ recocida a $500{ }^{\circ} \mathrm{C}$, crece drásticamente para radiación con longitudes de onda en el rango entre 360 y $425 \mathrm{~nm}$ aproximadamente. Este crecimiento va desde 30 hasta $70 \%$. Luego se mantiene casi constante con un ligero pico para longitud de $570 \mathrm{~nm}$ hasta $78 \%$. Es decir, responde mejor que las demás películas en la mayor parte del rango del espectro visible. 
Figura 3. (a) Gráfica de transmitancia versus longitud de onda para películas delgadas de $\mathrm{TiO}_{2}$ de diferentes temperaturas de sustrato. (b) Efecto de la temperatura de fabricación del sustrato sobre el borde de absorción de las películas a diferentes temperaturas de sustrato, se toma la referencia del espectro de $\mathrm{TiO}_{2}$ fabricado a $380{ }^{\circ} \mathrm{C}$ sin recocido.

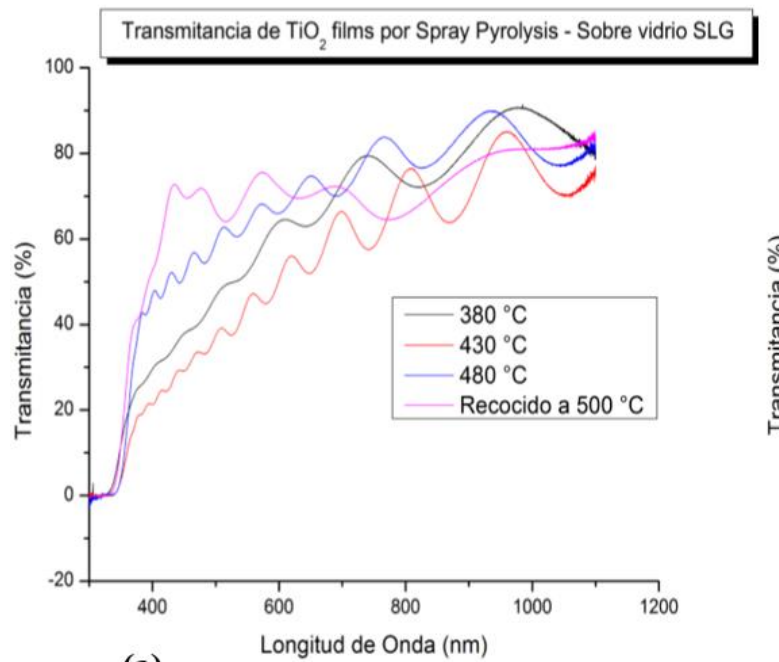

(a)

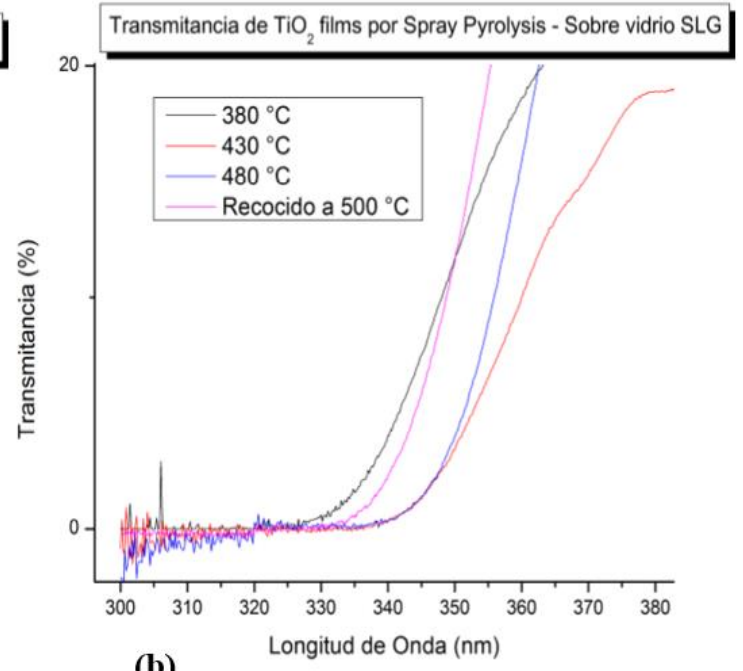

(b)

Las curvas de transmitancia de la figura 3a, presentan para todas las películas una fuerte caída de la transmitancia a longitudes onda por debajo de $400 \mathrm{~nm}$ (Shinde et al., 2008). Además, las películas fabricadas tienen prácticamente transmitancia nula ante radiación del espectro ultravioleta; a medida que la temperatura de sustrato crece, la capacidad de transmitir radiación con longitudes de onda perteneciente al espectro visible también se incrementa. La figura 3(b) muestra un acercamiento de la figura 3(a) en la zona de baja transmitancia. Tomando como referencia el espectro de transmitancia de la película de $\mathrm{TiO}_{2}$ fabricada a temperatura de $380{ }^{\circ} \mathrm{C}$ sin recocido (línea negra en Figura 3a), se observó que las demás películas de $\mathrm{TiO}_{2}$ obtenidas con mayores temperaturas de fabricación $\left(430{ }^{\circ} \mathrm{C}, 480{ }^{\circ} \mathrm{C}\right.$ y $380{ }^{\circ} \mathrm{C}$ con recocido a $500{ }^{\circ} \mathrm{C}$ ), experimentan un corrimiento en el borde de absorción del espectro de las películas, hacia longitudes de onda mayores conforme se incrementa la temperatura de fabricación en las mismas (Pérez, 2014).

\section{3 ÍNDICE DE REFRACCIÓN(N), ESPESOR DE LAS PELÍCULAS (T)}

En las figuras 4(a) y 4(b) se muestran los envolventes $T_{M}$ y $T_{m}$ en la zona de baja absorción. Los índices de refracción y los espesores fueron hallados utilizando los valores de estas envolventes de la zona B. El resumen del índice de refracción, el espesor y la porosidad de películas delgadas de $\mathrm{TiO}_{2}$, se puede apreciar en la tabla 2 . 
Tabla 2. Índices de refracción y espesor de películas delgadas de $\mathrm{TiO}_{2}$ obtenidas a diferentes temperaturas de sustrato en las zonas

$$
\text { Temperatura de sustrato }\left({ }^{\circ} \mathrm{C}\right)
$$

Índice de Refracción $\mathrm{n}_{\mathrm{p}}$

Espesor $\mathrm{t}(\mathrm{nm})$

Porosidad P (\%)

Ancho de banda prohibida $\mathrm{E}_{\mathrm{g}}(\mathrm{eV})$

$\begin{array}{ccc}380 & 430 & 480 \\ & & \\ 2,40 & 2,43 & 2,15 \\ 641,29 & 1095,00 & 1020,64 \\ 3,40 & 2,01 & 13,74 \\ 3,234 & 3,213 & 3,196\end{array}$

380 recocido a 500

1,96
731,95
23,39
3,204

1,96

23,39

3,204

Figura 4 (a). Espectro típico de transmitancia para películas de $\mathrm{TiO}_{2}$ fabricadas a $380{ }^{\circ} \mathrm{C} \mathrm{y} \mathrm{(b)} 430{ }^{\circ} \mathrm{C}$. Donde se muestra la zona de baja absorción (zona $\mathrm{B}$ ) con la finalidad de trazar las envolventes $\mathrm{T}_{\mathrm{M}}$ y $\mathrm{T}_{\mathrm{m}}$. (c). Espectro típico de transmitancia para películas de $\mathrm{TiO}_{2}$ fabricadas a $480{ }^{\circ} \mathrm{C}$ y (d) Con recocido a $500{ }^{\circ} \mathrm{C}$. Donde se muestra la zona de baja absorción (zona B) con la finalidad de trazar las envolventes $\mathrm{T}_{\mathrm{M}} \mathrm{y} \mathrm{T}_{\mathrm{m}}$.

(a)
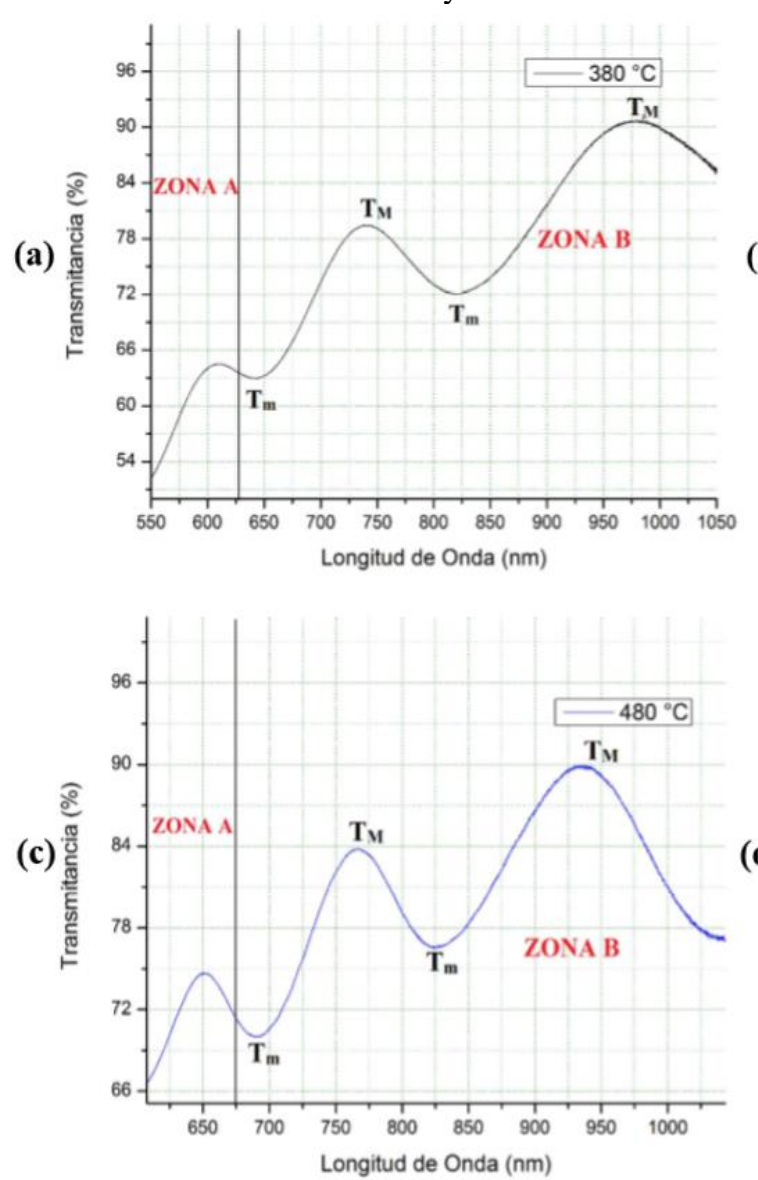

(b)
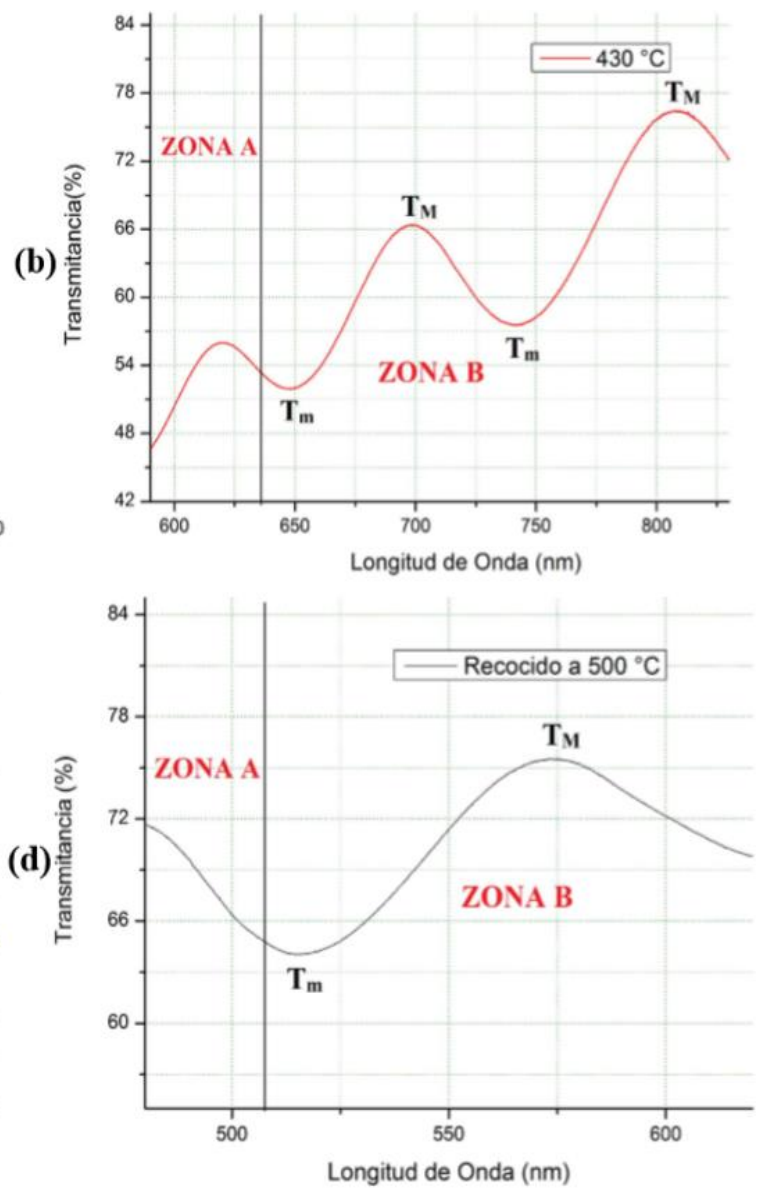

Se encontró que las películas presentan índices de refracción que se encuentra en el rango de 1,9 - 2,43 para espectros de baja transmitancia. Este rango se encuentra en el orden de lo reportado en la referencia para películas de $\mathrm{TiO}_{2}$ fabricadas por rocío pirolítico (Oja et al., 2004; Manoharan y Sridhar, 2012), por el método sol-gel (Hanini et al., 2013; Bartic et al., 2013) y por el método de deposición de alto vacío (Narasimha, 2002). Los resultados del último autor revelan valores de índice de refracción en el rango $2,19-2,32$. 
Respecto al espesor, se obtuvieron películas con espesores desde 1095,00 nm para películas con temperatura de fabricación de $430{ }^{\circ} \mathrm{C}, 1020,64 \mathrm{~nm}$ para películas con temperatura de sustrato de $480{ }^{\circ} \mathrm{C}$, $731,95 \mathrm{~nm}$ para aquellas películas con recocido a $500{ }^{\circ} \mathrm{C}$ y $641,29 \mathrm{~nm}$ para las temperaturas de substrato de $380^{\circ} \mathrm{C}$. Los valores hallados se encuentran en la escala de valores revelados en los trabajos en películas delgadas por rocío pirolítico realizados por (Castillo et al., 2004; Shinde et al., 2008). Pero no son cercanos a los valores revelados por (Acosta et al., 2005; Hanini et al., 2013). A pesar de no ser cercanos concuerda con (Acosta et al., 2005; Castillo et al., 2004) respecto a la misma tendencia a crecer mientras se incrementa la temperatura de sustrato. Por otra parte, la tabla 2 muestra al comienzo una ligera caída de la porosidad y luego un apreciable incremento de la misma a medida que aumenta la temperatura de sustrato de películas delgadas de $\mathrm{TiO}_{2}$. Estos resultados son prácticamente opuestos a los resultados encontrados por (Bartic et al., 2013; Hanini et al., 2013).

\subsection{ANCHO DE BANDA PROHIBIDA $\left(\mathrm{E}_{\mathrm{G}}\right)$}

En la figura 5(a) se presenta el grafico de $(\mathrm{h} v * \square)^{1 / 2}$ característico del modelo de Tauc, para transiciones indirectas, como una función la energía del fotón (hv). A partir de este gráfico se realizó la regresión lineal en la zona más recta de la curva, obteniendo la ecuación de ésta, donde la extrapolación nos permite obtener el valor del ancho de banda prohibida con los datos de la pendiente $(\mathrm{m})$ y la ordenada al origen (b) de la ecuación. Para determinar el valor de $\mathrm{E}_{\mathrm{g}}$; se asumió que (hv* $\left.\square\right)^{1 / 2}=0$, por lo tanto, $\mathrm{E}_{\mathrm{g}}$ $=-\mathrm{b} / \mathrm{m}$; como se muestra en la Figura 5(b). Para las películas fabricadas a $380{ }^{\circ} \mathrm{C}, 430{ }^{\circ} \mathrm{C}, 480{ }^{\circ} \mathrm{C}$ y 380 ${ }^{\circ} \mathrm{C}$ recocida a $500{ }^{\circ} \mathrm{C}$ se obtuvieron energías de banda prohibida de $3,234 \mathrm{eV}, 3,210 \mathrm{eV}, 3,196 \mathrm{eV}$ y 3,204 $\mathrm{eV}$ respectivamente

Figura 5 (a). Extrapolación de datos con tendencia lineal extraídos de la gráfica $(\alpha h v)^{1 / 2}$ vs h $v$ para determinar el ancho de banda prohibida de una película crecida a $480{ }^{\circ} \mathrm{C}$. (b) Gráfica $(\alpha \mathrm{h} v)^{1 / 2} \mathrm{vs} \mathrm{h} v$ y extrapolación para las películas de $\mathrm{TiO}_{2}$ fabricadas a (a) $\mathrm{T}_{\mathrm{s}}=380{ }^{\circ} \mathrm{C}$, (b) $\mathrm{T}_{\mathrm{s}}=430{ }^{\circ} \mathrm{C}$, (c) $\mathrm{T}_{\mathrm{s}}=480{ }^{\circ} \mathrm{C}$ y (d) $\mathrm{T}_{\mathrm{s}}=380{ }^{\circ} \mathrm{C}$ recocida a $500{ }^{\circ} \mathrm{C}$.

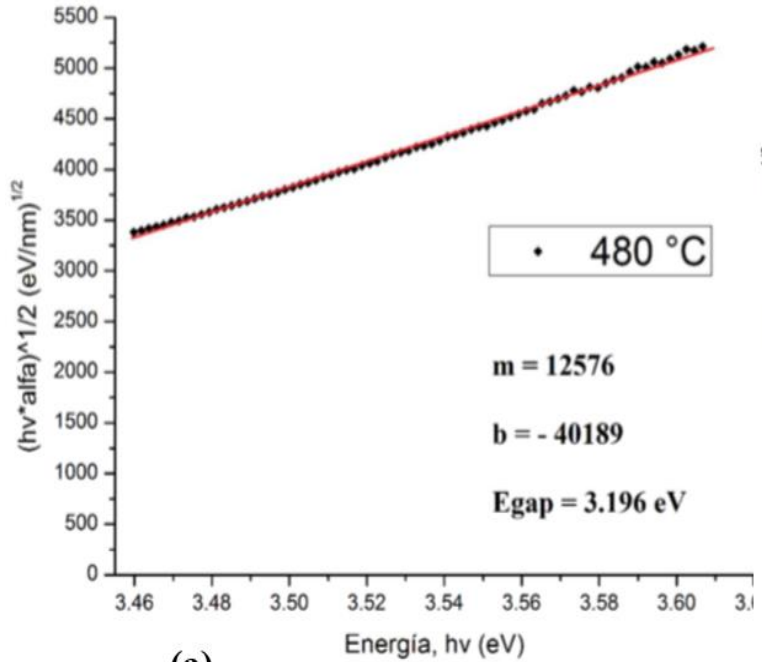

(a)

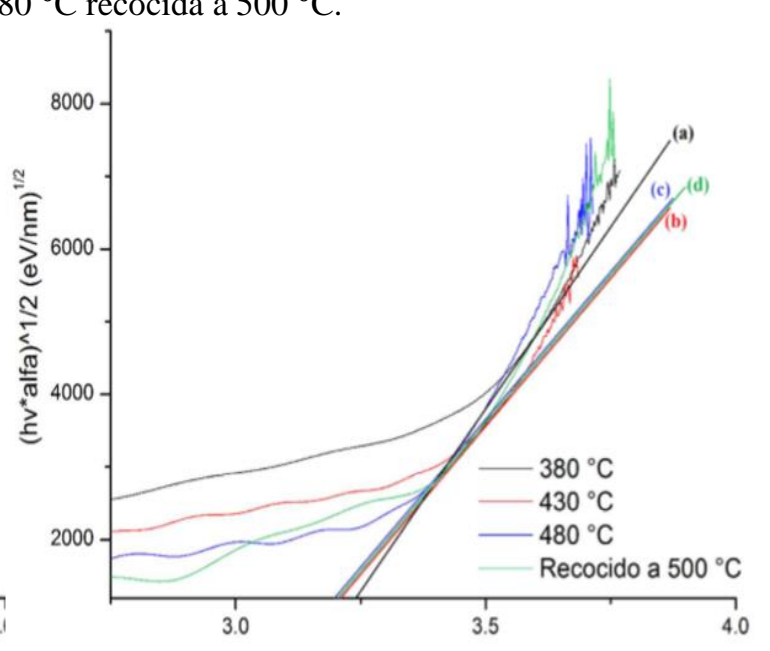

(b)
Energia, hv (eV) 
En esta tabla se aprecian valores cercanos a 3,20 eV con tendencia a disminuir mientras aumenta la temperatura en el caso de gaps pertenecientes a películas depositadas a 380; 430 y $480{ }^{\circ} \mathrm{C}($ Acosta $e t$ al., 2005; Manoharan y Sridhar, 2012; Hanini et al., 2013; Bartic et al., 2013; Gapale et al., 2018).Los valores del ancho de banda prohibida para las películas de $\mathrm{TiO}_{2}$ se muestra en la tabla 2 .

\subsection{GRÁFICA VOLTAJE - CORRIENTE}

Para las películas de 3 capas fabricadas a temperatura de sustrato de 380,430 y $480{ }^{\circ} \mathrm{C}$ no se muestran datos de corriente y voltaje aplicando al someterlas a medición por el método de cuatro puntas colineales debido a que el valor de la resistencia eléctrica es muy elevado y no se encuentra dentro del rango medible del instrumento. Dicho de otra manera; la intensidad de la corriente que atraviesa las películas podría estar por debajo del límite de medición del instrumento y, en consecuencia, su valor no pudo ser estimado correctamente (Bartic et al., 2013). La razón por la cual la resistencia de estas películas es elevada puede ser atribuida a la poca vacancia de oxígeno presente en la estructura (Pérez, 2014). Sin embargo, para aumentar la vacancia de oxígeno, se fabricaron películas con doble concentración y se incrementaron el número de capas hasta 10 y 9 con temperatura de sustrato de $480{ }^{\circ} \mathrm{C}$, obteniendo datos de corriente y voltaje los cuales gráficamente presentan unas características de contacto óhmico entre las películas delgadas y el contacto metálico de plata, a partir de 1,4 $\mu \mathrm{A}$ aplicados. Por lo tanto, el ajuste lineal de los datos obtenidos permitió obtener la pendiente de la recta que corresponde al valor de la resistencia eléctrica del material.

Figura 6. Gráfica de Voltaje vs Corriente de muestras fabricadas a $480{ }^{\circ} \mathrm{C}$ a doble concentración con 9 y 10 capas donde se muestran sus tendencias lineales.
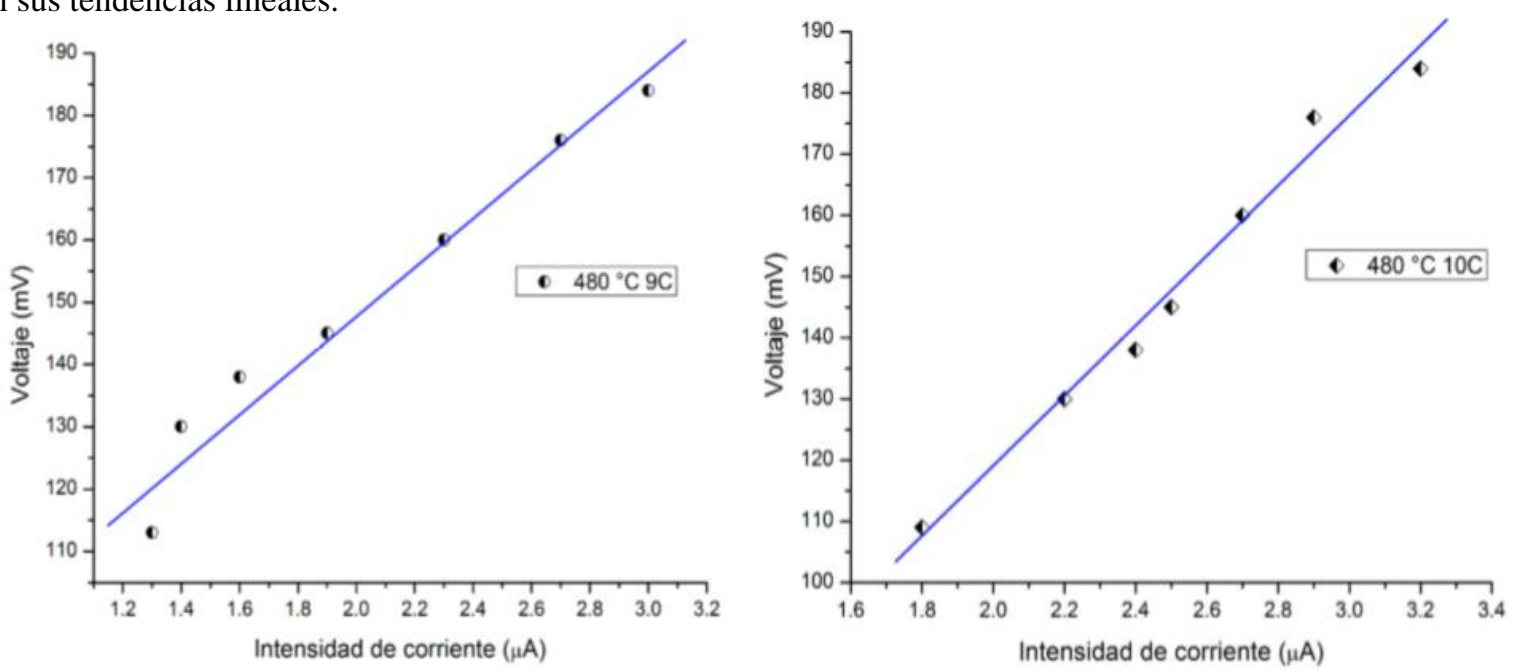

Se debe tomar en cuenta que, para hacer las mediciones de voltaje y corriente, las películas no fueron expuestas a ningún tipo de excitación ultravioleta. Simplemente fueron medidas al aire a 
temperatura ambiente en ausencia de radiación UV. La muestra de 9 capas, a partir de 1,4 $\mu \mathrm{A}$ aplicados, tiene comportamiento lineal y la pendiente de la gráfica corresponde a la resistencia eléctrica cuyo valor es de $38 \mathrm{~K} \Omega$ aproximadamente. Por otra parte, la muestra de 10 capas muestra también un comportamiento lineal cuyo valor de pendiente viene a ser su resistencia eléctrica, el cual ronda los $57 \mathrm{~K} \Omega$. Se puede notar que el número de capas influye en las características de voltaje - corriente.

\section{CONCLUSIONES}

De acuerdo a los resultados obtenidos, a $380{ }^{\circ} \mathrm{C}$ de temperatura de sustrato, las películas de $\mathrm{TiO}_{2}$ son totalmente amorfas. Sin embargo, el aumento de la temperatura de deposición o posteriores tratamientos térmicos mejoran la oxidación de la película contribuyendo a la difusión de los átomos a través de la red la cual presenta fase anatasa a partir de $430{ }^{\circ} \mathrm{C}$. Incluso a $480{ }^{\circ} \mathrm{C}$ se muestra un pico $(211)$ de rutilo dando inicio a la formación de estados de transición de fase anatasa - rutilo. Cuando se trata de fabricación de películas delgadas de $\mathrm{TiO}_{2}$ por rociado pirolítico, la presencia de fase rutilo a $480{ }^{\circ} \mathrm{C}$ es un hecho poco común o no está reportado en la literatura, la cual suele presentar picos de rutilo para temperaturas de sustrato o de recocido por encima de los $600{ }^{\circ} \mathrm{C}$. Es un hecho poco conocido en la fabricación de películas delgadas por rociado pirolítico, pero se da como resultado de otros métodos de fabricación como ablación laser y deposición por arco catódico. El tamaño de grano de las películas presenta una tendencia a disminuir con la temperatura de sustrato y presenta su valor más pequeño a 480 ${ }^{\circ} \mathrm{C}$ el cual es $30,79 \mathrm{~nm}$. La transmitancia cae significativamente para radiaciones menores a $400 \mathrm{~nm}$ llegando a hacerse nula en el rango UV. La transmitancia mejora a medida que la temperatura de sustrato aumenta; tal es así que llega a presentar hasta $75 \%$ en el rango visible.

El índice de refracción de las películas delgadas de $\mathrm{TiO}_{2}$ tiende a disminuir con la temperatura de sustrato; mientras la porosidad aumenta. Se observó que la energía de banda prohibida disminuye desde 3,234 hasta 3,196 eV al aumentar la temperatura de sustrato. Esto indica que el material mejora su cristalinidad. El espesor de las películas tiende a disminuir para valores de temperatura de sustrato por encima de $430{ }^{\circ} \mathrm{C}$. 


\section{REFERENCIAS}

Acosta, Dwight R., Martínez, Arturo I., López, Alcidez A., Magaña, Carlos R. 2005. Titanium dioxide thin films: the effect of the preparation method in their photocatalytic properties. Journal of Molecular Catalysis A: Chemical, 228, $183-188$.

Agudelo Morimitsu L. C., Escobar Rincón, D., De La Roche Yepes, J., Restrepo Parra E., Arango Arango, P. J. 2014. Producción de películas delgadas de TiO2 obtenidas por la técnica de arco catódico, Scientia et Technica Año XIX, Vol. 19, No. 1, Universidad Tecnológica de Pereira. ISSN 0122-1701.

Castillo, N., Olguín, D., and Conde-Gallardo, A. 2004. Structural and morphological properties of $\mathrm{TiO}_{2}$ thin films prepared by spray pyrolysis. Revista Mexicana de Física, 50 (4), 382 - 387.

Cullity, B. D. 1978. Elements of X - Ray Diffraction, 2nd. Ed., Addison Wesley Publishing Company, Inc. USA, pp. $81-100$.

Escobar Alarcón L., Haro Poniatowski E., Camacho López M.A., Fernández Guasti M., Jiménez Jarquín J., Sánchez Pineda A. 1999. Structural characterization of TiO2 thin films obtained by pulsed laser deposition. Applied Surface Science 137, pp. 38 - 44.

Franco, L. 2018. Estudio y optimización del crecimiento de films de $\mathrm{TiO}_{2}$ por arco catódico para aplicaciones en biomateriales. Tesis de doctorado, Universidad de Buenos Aires, Buenos Aires. Argentina. $171 \mathrm{pp}$.

Goodman A. M. 1978. Optical interference method for the approximate determination of refractive index and thickness of a transparent layer; Applied Optics; 17, 2779-2787.

Gapale, DL.; Borse, RY. 2018. Effect of temperature on structural, optical and electrical properties of spray deposited $\mathrm{TiO}_{2}$ thin films. Int. Res. J. of Science \& Engineering; Special Issue A2: 116 - 121.

Hanini F., Bouabellou A., Bouachiba Y., Kermiche F., Taabouche A., Hemissi M., and Lakhdari D. 2013. Structural, optical and electrical properties of $\mathrm{TiO} 2$ thin films synthesized by sol-gel technique. IOSR Journal of Engineering (IOSRJEN) e-ISSN: 2250-3021, p-ISSN: 2278-8719 Vol. 3, Issue 11. V1, pp. 2128.

Kumar, R.; Sharma, N.; Arora, N. 2016. Study of spray deposited titanium dioxide films. Advances in Applied Science Research 7(3): 142 - 147.

Kang, H.S., Sohn, J.R., Kang, Y.C., Jung, K.Y., Park, S.B. 2005. The characteristics of nano-sized Gddoped $\mathrm{CeO} 2$ particles prepared by spray-pyrolysis. Journal of Alloys and Compounds. 398, pp. 240 - 244.

Manoharan, C. and Sridhar, R. 2012. Physical properties of spray pyrolysed TiO2 films. International Journal of Recent Scientific Research Vol. 3, Issue, 9, pp.775 -777. 